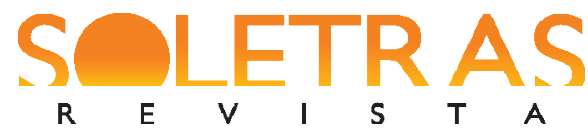

N. $40-2020.2$ - ORNA LEVIN

MARIE-ÉVE THÉRENTY

\title{
Entretien avec le Professeur Docteur Marie-Ève Thérenty
}

Por

Orna Messer Levin ${ }^{1}$

Marie-Ève Thérenty graduou-se pela École Normale Supérieur Fontenay/Saint-Cloud (ENS), fez Mestrado em Letras Modernas na Universidade Paris VII (Denis Diderot) sob orientação de Francis Marmade, e obteve o Diploma de Especialização em história, ciência política e ciência da informação/comunicação pelo Institut dấtudes Politiques de Paris (IEP). Concluiu o Doutorado na Universidade Paris VII (Denis Diderot) defendendo a tese ñMosaïque, être écrivain entre presse et roman (1829-1836)ò, sob orientação de Jean Delabroy. É professora e pesquisadora da Universidade Monpellier III (Paul Valéry), onde dirige, desde 2008, o grupo RIRRA 21 (ñReprésenter, Inventer la Realité du Romantisme à l'Aube du XIX siècleò). Coordenou a equipe francesa de investigadores do projeto Médias19, desenvolvido em parceria com universidades do Québec, o qual foi agraciado com financiamento das agências ANR e FQRSC. Atualmente é responsável pelo Numapresse (ñPresse et Humanités Numériquesò), projeto subvencionado pela ANR, e integrante do projeto de cooperação entre bibliotecas europeias e instituições universitárias intitulado NewEye: a Digital Investigator for Historical Newspapers. Participou da organização de nove publicações coletivas, dentre as quais o livro premiado Civilisation du journal. Histoire culturelle et littéraire de la presse française du XIX siècle (2012), o volume Presse et plumes. Journalisme et littérature au XIX ${ }^{e}$ siécle (2004) e o recente L'écrivain comme marque (2018). Em sua vasta produção acadêmica, constam mais de uma centena de artigos e sete livros autorais a propósito das pesquisas sobre literatura e imprensa, tais como La Littérature au quotidien. Poétiques journalistiques au dix-neuvième siècle (2007) e Femmes de presse, femmes de letrres. De Délphine de Girardin à Florence Aubenas (2019).

Soletras: Pourriez-vous nous parler de votre parcours professionnel ? Qu@̂est-ce qui a motivé votre intérêt académique pour la presse?

Thérenty: Au début de ma carrière, avant la soutenance de ma thèse, jâai été tentée par les milieux de la culture et du journalisme. Jâi donc travaillé comme chargée de mission auprès du président de lấtablissement public de la Bibliothèque Nationale de

\footnotetext{
${ }^{1}$ Professora Livre Docente pela Unicamp atua na área de literatura brasileira junto ao Programa de PósGraduação em Teoria e História Literária (PGTHL), Instituto de Estudos da Linguagem, da Unicamp. Email: orna@unicamp.br
} 


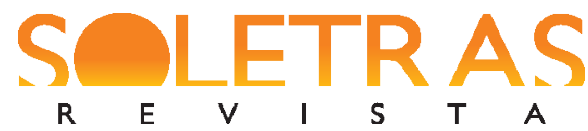

France, jâii exercé aussi comme tout bon écrivain du XIX ${ }^{\mathrm{e}}$ siècle, des missions de polygraphe dans plusieurs maisons dấdition où jâii rédigé beaucoup dôuvrages de vulgarisation, des dictionnaires, tout en enseignant un peu dans le secondaire et dans le supérieur. Après la soutenance de ma thèse en 2000, jôai obtenu un poste de maître de conférences à lâıniversité Paul Valéry Montpellier 3 et jâii véritablement découvert ma voie. Etre chercheur à lâıniversité permet dệxercer plusieurs métiers : professeur, chercheur, écrivain, organisateur dấvénements culturels, administrateur même, si lôn en éprouve le désir (ce qui nâst pas mon cas). Depuis 2007, je suis professeure dans cette université qui mâ tout donné et je dirige un centre de recherche, le RIRRA21, spécialisé dans les relations entre littératures, médias et arts, qui permet de travailler en collectivité. Jâi obtenu aussi plusieurs financements importants qui mônt permis de conduire tranquillement mes recherches sur la presse.

Mon intérêt académique pour la presse vient de mon milieu familial. La famille de ma mère est une famille dấcrivains journalistes dont certains ont fait une belle carrière. Mon grand-père était à la fois un dix-neuviémiste qualifié, un écrivain et il a été aussi pendant un temps un très grand critique littéraire. Je crois que je fais lâensemble de ce cheminement pour arriver à écrire son histoire durant la Seconde guerre mondiale.

Soletras: Au Brésil, la presse fait souvent lôbjet de recherches de la part des historiens et, dans une moindre mesure, des chercheurs en littérature. Cela est-il également le cas en France?

Thérenty: Longtemps en France la recherche sur la presse a été effectivement réservée aux historiens et notamment aux historiens du politique. Ils ont balisé le champ, réalisant un travail important grâce à des monographies mais aussi à des grandes entreprises collectives comme lô̂Histoire générale de la presse française publiée aux PUF.

Les spécialistes du XVIII ${ }^{\mathrm{e}}$ siècle, Jean Sgard, Pierre Rétat et Claude Labrosse, ont enclenché ensuite un mouvement de resaisie de cette histoire par les littéraires, bientôt suivi par les dix-neuviémistes (Roger Bellet, Jean-Claude Vareille, Lise Dumasy) qui ont renouvelé lôhistoire de la presse. La publication ensuite par une équipe dôhistoriens 


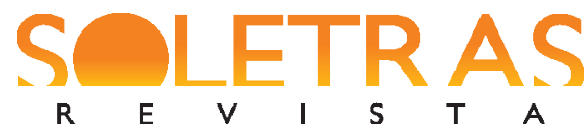

et de littéraires de La Civilisation du journal. Histoire culturelle et littéraire de la presse au dix-neuvième siècle (nouveau monde éditions, 2011) a, je crois, complètement refondé lôhistoire des études de presse. Lôhypothèse de liaisons et de corrélations entre lôhistoire littéraire et lôhistoire de la presse, entre poétique de presse et littérature a été la source dônn nombre important de travaux et de doctorats littéraires ces dernières années qui ne se sont pas cantonnés au $\mathrm{XIX}^{\mathrm{e}}$ siècle mais qui se sont étendus au $\mathrm{XX}^{\mathrm{e}}$ siècle. Plusieurs monographies ont souligné lômportance de la matrice littéraire dans lấlaboration de lấcriture journalistique. Parallèlement, les genres journalistiques (la chronique, le fait divers, le reportage, lônterview) ont fait lôbjet de nombreux travaux publiés. Les études de presse sont aujourdôui un courant extrêmement dynamique des études de presse littéraires, sans doute renforcé par la collaboration internationale entre plusieurs équipes. Un projet comme Numapresse (numapresse.org) que je dirige réunit outre des historiens et des littéraires des spécialistes de sciences de lônformation et de la communication et des spécialistes dôumanités numériques.

Soletras: À partir de vos études sur la littérature dans les périodiques, considérez-vous pertinente la distinction de valeur, souvent présente dans les études littéraires, entre les î uvres du canon et les textes littéraires dits mineurs ou éphémères?

Thérenty: Câsst une question délicate. Je pourrais tenter de lấsquiver en faisant remarquer que la plupart des oeuvres du canon au $\mathrm{XIX}^{\mathrm{e}}$ siècle et durant le premier $\mathrm{XX}^{\mathrm{e}}$ siècle sont en fait parues dôbord dans les journaux. Cela vaut pour les romans aussi bien que pour la poésie ou le théâtre comme lôa montré la thèse récente dôAmélie Calderone. Les textes du canon sont souvent ceux qui ont lôhonneur dône édition en librairie tandis quâffectivement ceux qui ne sont pas repris sont considérés comme mineurs. Pourtant une analyse poétique montre que les deux corpus répondent aux mêmes contraintes. Nous sommes victimes, il faut le reconnaître, dồne conception de la littérarité qui sâst imposée avec la modernité et qui voit dans lâutonomie, lôntransitivité, la gratuité les critères de la littérature. Dans ce cadre les textes des journaux parce quôls sont souvent explicitement reliés à la réalité et à lôactualité paraissent souvent inférieurs. Je ne tranche pas, en fait. Il faut voir au cas par cas. Je ne 


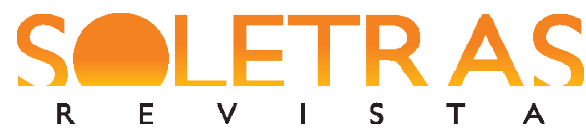

me voue pas en tout cas à une réhabilitation systématique des î uvres mineures mais en même je fais remarquer lôhistoricité de la notion de valeur. Il me semble évident par exemple que la considération académique aujourdôui de la littérature médiatique est liée aux changements culturels contemporains, à la progression de la sérialité comme paradigme majeur, à la croissance des fictions industrielles. Câst parce que la définition de la littérature est en train de vriller du fait de nos expériences culturelles communes et globales que les textes périodiques sont soudain devenus des sujets dấtudes et de recherches.

Soletras: Au Brésil, il est courant que les chercheurs en littérature se tournent vers les journaux et revues afin de retrouver les textes que les auteurs consacrés y avaient lancés avant de les faire publier en format livre. Ainsi, fautes dôarchivage, les premières versions retrouvées dans la presse remplaceraient-elles les manuscrits inexistants ou égarés. Que pensez-vous de ce type de démarche?

Thérenty: La situation est sans doute différente en France où au $\mathrm{XIX}^{\mathrm{e}}$ siècle, les écrivains ont commencé à conserver leurs manuscrits, voire parfois même à les monnayer ou à les offrir. Il est donc fréquent dôvoir pour les écrivains à la fois des manuscrits, des versions publiées dans les journaux puis en librairie. Selon moi en tout cas, les versions publiées dans les journaux ne sont pas exactement des prépublications ou des brouillons. Les î uvres sont contraintes par leurs lieux de publication et donc lâensemble de la littérature du $\mathrm{XIX}^{\mathrm{e}}$ siècle est liée aux formats et aux dispositifs imposés par les journaux. Il faut donc les considérer comme un maillon essentiel de la chaîne de production et ne pas en faire des avant-textes.

Soletras: Quelles contributions lâxamen des périodiques a-t-il apporté à lônterprétation des textes et à la connaissance des genres littéraires?

Thérenty: Cette question massive pourrait renvoyer à lânsemble de mes travaux. Je vais répondre donc autrement. Je crois que le fait que lôhistoire littéraire ait longtemps 


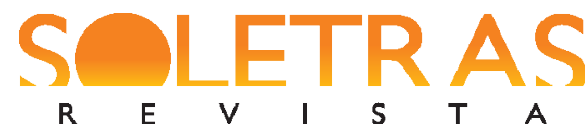

oublié de considérer la question des supports et notamment la question médiatique a entraîné, sinon des contresens, au moins des lacunes essentielles dans la connaissance des genres et des î uvres depuis le XVIII ${ }^{\mathrm{e}}$ siècle. Comment par exemple analyser le réalisme sans prendre en compte le fait que les écrivains du XIX ${ }^{\mathrm{e}}$ siècle publiaient leurs romans dans des journaux dans une rubrique contiguë avec les rubriques des faits divers, des débats parlementaires ou judiciaires ? Comment comprendre lônvention du poème en prose sans envisager la chronique journalistique ? $\mathrm{Au} \mathrm{XIX}^{\mathrm{e}}$ et pendant une grande partie du $\mathrm{XX}^{\mathrm{e}}$ siècle, la plupart des écrivains publiaient leurs $\hat{\imath}$ uvres dans les journaux et par ailleurs pour des raisons alimentaires mettaient leur plume au service de lấcriture dônformation pour des chroniques ou des reportages. Lôhistoire littéraire française sâst heureusement employée depuis quelques années dôabord à rééditer de manière systématique lỗ uvre journalistique de ses écrivains puis à produire des essais qui ont fleuri de manière régulière sur cette production. On a dôabord eu un Vallès journaliste (par Roger Bellet), puis un Balzac journaliste (par Roland Chollet), un Zola journaliste (par Henri Mitterand)é Tous ces essais ont déclenché ensuite une relecture de lâensemble de lẫ uvre de ces écrivains à lâune du journalisme. Quant aux genres littéraires, ils sont aussi soumis à cette vague de relectures. En témoigne, un exemple parmi dôutres, le bel essai de Marie-Astrid Charlier Le Roman et les jours (Garnier, 2018) qui relit lânsemble du corpus romanesque du $\mathrm{XIX}^{\mathrm{e}}$ siècle à la lumière de la nouvelle injonction temporelle imposée par le journal: la quotidienneté. Elle montre que lấcriture réaliste du quotidien souvent réduite à une écriture prosaïque du détail est en fait une nouvelle appréhension temporelle de la réalité imposée par lâxpérience de la quotidienneté véhiculée par le journal.

Soletras: Lấtude des régimes dấcriture littéraire dans la presse est, dôıne certaine façon, une nouveauté et ouvre de nouveaux champs de recherches dans les domaines de la littérature et de lôistoire. La culture médiatique, apparue au XIX siècle, a-t-elle laissé son héritage à la culture du XXI siècle?

Thérenty: Les exemples sont évidemment nombreux puisque la culture du $\mathrm{XXI}^{\mathrm{e}}$ siècle notamment celle qui sômpose avec les séries, les jeux vidéo, les bandes dessinéesé est 


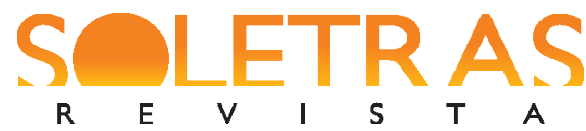

une directe héritière de la culture médiatique. Les premiers historiens de la culture ont tracé cette généalogie en étudiant le fil qui relie le roman-feuilleton du XIX ${ }^{\mathrm{e}}$ siècle aux grandes fictions industrielles mondiales contemporaines de Game of Thrones à Matrix. Avec une petite équipe nous avons essayé dans une série de travaux de montrer le lien qui unit la circulation mondiale des mystères urbains au $\mathrm{XIX}^{\mathrm{e}}$ siècle à la suite de la parution des Mystères de Paris dôEugène Sue en 1842 dans le Journal des débats et la diffusion globale aujourdôui de grandes fictions parfois qui semblaient au départ périphériques comme La Casa de Papel. Celui qui incontestablement trace le mieux cette histoire et montre la naissance de la culture sérielle au $\mathrm{XIX}^{\mathrm{e}}$ siècle et ses prolongations aujourdônui avec les fictions transmédiatiques est Matthieu Letourneux. Je renvoie à son excellent livre, Fictions à la Chaîne (Seuil, 2017). Câst lâxxemple le plus massif mais évidemment beaucoup de chantiers de recherche plus petits procurent de grands plaisirs de découvertes. Nous avons fait la généalogie qui mène de lấpigramme de presse aux formes brèves sur internet par exemple. Nous avons montré comment la culture satirique de Charlie Hebdo trouvait son origine dans les écritures de la petite presse du $\mathrm{XIX}^{\mathrm{e}}$ siècle. Surtout dans un livre très récent intitulé Fake news et viralité avant internet et signé Roy Pinker (CNRS, 2020), nous sommes revenus sur le fait que la culture de la fake news, du mème et du placement produit sấtait inventée dès lântrée dans lầre médiatique, bien avant la naissance dânternet.

Soletras: La diffusion mondiale de la culture française au XIX siècle a été rendue possible grâce à des réseaux efficaces dôagents composés dômprimeurs, de libraires ou encore dốditeurs. La traduction et la diffusion de la littérature française au sein des journaux locaux dans de nombreux pays ont également joué un rôle fondamental dans la diffusion de la littérature française à lấtranger. Selon vous, les spécialistes de la littérature française canonique prennent-ils en compte dans leurs recherches la diffusion transnationale des î uvres littéraires françaises par les traductions et leur circulation en dehors de lôHexagone?

Thérenty: Il sâgit dôun champ de recherche très dynamique. Mais je crois honnête de remarquer que les spécialistes de la littérature française canonique sont souvent moins 


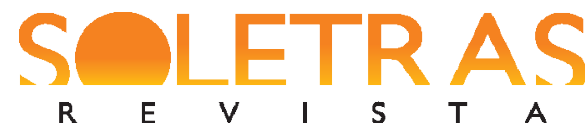

intéressés que les spécialistes de littérature populaire par la circulation globale des î uvres. Jôi parlé un peu plus haut de ce projet que nous avions mené et dont les actes figurent sur Medias19.org sur la circulation/traduction des mystères urbains. Les chercheurs qui étudient la circulation du canon sont donc souvent des historiens (Blaise Wilfert), des sociologues (Gisèle Sapiro) ou encore très souvent des universitaires étrangers spécialistes de la littérature française qui étudient la circulation/traduction de Zola, de Balzac ou de Sand par exemple dans leurs propres pays.

Soletras: Dans votre dernier livre sur les écrivaines journalistes, vous vous êtes penchée sur la constitution de lôdentité féminine. Dâaprès vos recherches, serait-il possible dôaffirmer lôexistence dôın mode dốcriture féminin?

Thérenty: Je ne vais parler que pour le journalisme qui est vraiment mon domaine de spécialité. Je pense que longtemps il nô a pas eu un mode dấcriture journalistique féminin mais des modes dấcritures journalistiques féminins. Mais attention, il ne faut pas se méprendre sur mes propos. Il ne sâgit pas ici de postuler des essences sexuées. Pour comprendre pour quelles raisons les femmes journalistiques ont eu besoin de se trouver des modèles emblématiques, de se créer des postures spécifiques, de se chercher des lieux caractéristiques et des poétiques propres, il faut revenir au caractère social de lấcriture journalistique. Elle est dâbord témoignage, restitution dồn certain rapport au réel ; elle est ensuite une activité économique, rétribuée, qui sêxerce dans des structures dâentreprise; elle est enfin un travail exercé dans le cadre dôıne collectivité et de réseaux. Dans ce cadre, les femmes présentent plusieurs particularités qui ont longtemps distingué leur activité journalistique de celle des hommes, au moins jusquân 1944. Leur socialisation, c@̂st-à-dire la transmission de valeurs, de normes, de règles organisée par lấcole, la famille, lấglise est évidemment spécifique. De plus, leur statut légal tel que lâ défini le code Napoléon est celui de mineures. Il leur manque un certain nombre de droits essentiels, notamment dans le cas de la femme mariée, comme par exemple la liberté du choix du métier ou des études, lôndépendance des ressources, ce qui non seulement entrave leur activité mais dônne manière plus insidieuse entraîne un rapport au monde totalement différent. Elles sont subalternes dans la société, elles ne 


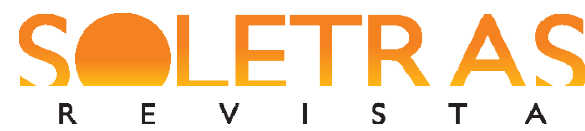

sont pas légitimes dans certains lieux publics comme les universités, les académies ou les espaces politiques. Et même si progressivement, entre 1848 et 1940, certains interdits se lèvent, le facteur principal qui fondamentalement exclut toute possibilité de traiter leur activité journalistique comme similaire à celle des hommes est le fait que jusquận 1944, elles nônt ni le droit de vote ni celui de se faire élire à des postes importants dans cette sphère publique dont le journal rend compte. A partir de là, comment même imaginer quêlles puissent rendre compte du réel de la même façon que les puissants et les établis ? Comment imaginer que leur field positioning (conditions dôccès à la profession, statut professionnel, habillements, droits politiques) très différent de celui des hommes, nâit pas dônfluence sur leur textual positioning (le ton, la voix, lâısage de la performativité, le rapport à lôbjectivité, le choix des rubriques, des postures et des points de vue )?

Mon hypothèse est que le journalisme des femmes françaises a été dôutant plus inventif quâl était contraint et que sa méconnaissance, liée à la fabrique, au vingtième siècle, dôıne histoire de la presse française peu intéressée par la question du genre (masculin/féminin) et dôilleurs toujours conduite par des hommes, a nui à une juste appréciation de leur place dans le paysage périodique. Mon livre participe donc à la fois dôın renouvellement de lôhistoire de la presse mais aussi de la considération de lôhistoire des femmes qui sâffirme depuis une vingtaine dônnées dans tous les champs sous lômpulsion des gender studies américaines. Une fois posé ce cadre, jâdentifie effectivement dans lôhistoire non pas un mais six modèles dấcritures journalistiques au féminin.

Soletras: Pensez-vous quâll existe encore un terrain fertile pour le développement de nouveaux projets sur la littérature et la presse?

Thérenty: En fait, les possibilités de recherche paraissent infinies et considérablement accrues par les progrès techniques en matière de numérisation. Dâbord les études médiatiques françaises doivent sôntéresser au $\mathrm{XX}^{\mathrm{e}}$ siècle. La Civilisation du journal sârrêtait en 1914 mais peut-être avons-nous été victimes ici de catégories chronologiques prépensées par lâıniversité française car cette hybridation entre 


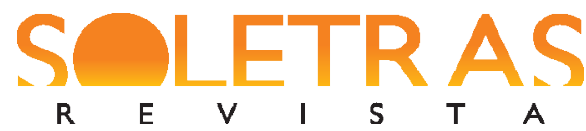

N. $40-2020.2$ - ORNA LEVIN

MARIE-ÉVE THÉRENTY

journalisme et littérature perdure en France jusquâ la Seconde Guerre mondiale au moins pour les journaux quotidiens et bien au-delà pour les magazines, les hebdomadaires, les fanzines comme le montrent des travaux récents. Certains quotidiens comme Paris-Soir ou Ce Soir, le journal dôAragon, attendent leurs grandes études de poétique historique tout comme la série des hebdomadaires des années trente qui constituent une mine dôıne richesse inouïe et inexplorée : Vendredi, Voilà, Confidences, Candide, Gringoireé Je rêve aussi dône grande étude littéraire sur le journal Libération depuis sa fondation sous lấgide de Jean-Paul Sartre en 1973 jusquâ aujourdôui. Par ailleurs, beaucoup des oeuvres des écrivains-reporters des années 1930 sont encore mal connues, y compris celles des plus grands comme Louis Béraud ou Andrée Viollis. La poétique médiatique nân est quầ ses débuts mais elle va engendrer des recherches passionnantes en montrant comment se transforme la réception dôın texte édité sur plusieurs supports depuis le journal jusquâu recueil éditorial en passant par le réinvestissement romanesque. Lôeuvre de J. Kessel serait ainsi particulièrement intéressante à étudier sous cet aspect. Des travaux aussi sont à affiner sur les sociabilités, sur les contraintes économiques et médiatiques qui pèsent sur les écrivains de lântre-deux guerres. Dans la littérature contemporaine, les oeuvres de certains écrivains français (Jean Rolin, Emmanuel Carrère, Jean Hatzfeldé ) tout comme certains périodiques de forme nouvelle à lônstar des mooks (la revue XXI ou Feuilleton) pourraient sênvisager avantageusement dans la perspective généalogique et méthodologique des rapports entre presse et littérature.

Mais surtout le développement des humanités numériques sur des corpus numérisés permet dôuvrir de nouveaux chantiers comme le montrent le développement du projet Numapresse (voir www.numapresse.org) et les outils extraordinaires que met en place Pierre-Carl Langlais, le chercheur sans doute le plus qualifié sur ces questions. La numérisation des corpus et le montage de scripts permettent par exemple dâxxtraire automatiquement des corpus de presse numérisés, des reportages, des romansfeuilletons ou des chroniques judiciaires (voir lôutil générothèque) et donc de travailler sur des corpus exhaustifs beaucoup plus rapidement. Les outils mis en place par Numapresse permettent aussi de penser à nouveau frais les questions de viralité et notamment de circulation et de reprise dôrticles et dôeuvres à échelle mondiale. Il est donc possible maintenant de suivre le devenir dônn fait divers qui sâest passé en France 


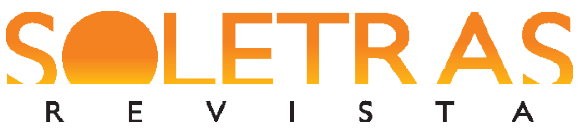

$\begin{array}{llllllll}\mathbf{R} & \mathbf{E} & \mathbf{V} & \mathbf{I} & \mathbf{S} & \mathbf{T} & \mathbf{A} & \text { N. } 40-2020.2-\text { ORNA LEVIN }\end{array}$

MARIE-ÉVE THÉRENTY

et qui va circuler dans le monde entier jusquầ reparaître sous la forme dồn conte en

Australie sous une autre signature.

Entretien tenu le 20 juillet 2020. 